\title{
Standardization of Iridium-192 Gamma-Ray Sources in Terms of Exposure
}

\author{
T. P. Loftus \\ Center for Radiation Research, National Bureau of Standards, Washington, D.C. 20234
}

September 29, 1979

\begin{abstract}
Iridium-192, in the form of small platinum-or stainless-steel-clad seeds, is used for radiation therapy. Standardization of this radionuclide, for the quantity exposure was carried out by measuring groups of seeds in an open-air geometry, using the NBS standard graphite cavity ionization chambers, and transferring the exposure data to a re-entrant ionization chamber.

Tables are provided from which the corrections for the graphite chamber have been calculated along with corrections for room scattering.

Radiographs of the source arrays are shown and details of the re entrant chamber source measurements and construction are provided.

As assessment of the errors involved in establishing this standard leads to a statement of 2 percent for the overall uncertainty in the calibration of an iridium seed for the quantity exposure.
\end{abstract}

Key words: Exposure standard; iridium-192 seeds; NBS standard graphite chambers; open-air geometry; reentrant chamber.

\section{Introduction}

The radionuclide iridium-192 has been used for radiation treatment of tumors for many years. Often the sources are manufactured by encapsulating short lengths of iridium-

TABLE 1. Dimensions and materials for the two types of iridium-192 seeds used for standardization measurements.

\begin{tabular}{lcc}
\hline \hline & $\begin{array}{c}\text { Alpha-Omega } \\
\text { Services, Inc. }\end{array}$ & $\begin{array}{c}\text { Rad-Irid } \\
\text { Inc. }{ }^{\mathrm{a}}\end{array}$ \\
\hline Wall: & Platinum & Stainless Steel \\
$\quad$ Material & $0.5 \mathrm{~mm}$ & $0.5 \mathrm{~mm}$ \\
Outside diameter & $0.1 \mathrm{~mm}$ & $0.2 \mathrm{~mm}$ \\
Wall thickness & $2.8-3.0 \mathrm{~mm}$ & $3.0 \mathrm{~mm}$ \\
Length & & \\
Source: & $10 \% \mathrm{Ir}-90 \% \mathrm{Pt}$ & $30 \% \mathrm{Ir}-70 \% \mathrm{Pt}$ \\
$\quad$ Composition & $0.3 \mathrm{~mm}$ & $0.1 \mathrm{~mm}$ \\
$\quad$ Diameter & $56 \mathrm{MBq}(1.5 \mathrm{mCi})$ & $56 \mathrm{MBq}(1.5 \mathrm{mCi})$ \\
$\quad$ Activity (nominal) &
\end{tabular}

a Certain trade names and company products are identified in order to adequately specify the experimental procedure. In no case does such identification imply recommendation or endorsement by the National Bureau of Standards, nor does it imply that the products are necessarily the best available for the purpose. alloy wire in stainless steel or platinum tubing for activation, and, because of their small size, the sources are called "seeds." Descriptions of the iridium-192 seeds used for the measurements described in this article are given in table 1.

Requests for standardization of this radionuclide in terms of exposure have been received at the National Bureau of Standards (NBS) on several occasions and, in collaboration with the companies supplying the sources to the medical community, work toward that end was begun early in 1977.

\section{General Considerations}

An iridium-192 source of $56 \mathrm{MBq}(1.5 \mathrm{mCi})$ activity produces an exposure rate (neglecting self absorption and air attenuation) of about $55 \mathrm{pA} / \mathrm{kg}(0.21 \mathrm{R} / \mathrm{s})$ at one meter. This value is calculated from the data given in table 2 and the following equation for the exposure rate [1]. ${ }^{1}$

$$
\dot{X}=\frac{A}{4 \Pi l^{2}(W / e)} \quad \sum_{i} P_{i} E_{i}\left(\mu_{e n} / \varrho\right)_{i}
$$


TABLE 2. Data for exposure-rate calculations for $3.7 \times 10^{10} \mathrm{~Bq}(1 \mathrm{Ci})$ of iridium-192. The data for $E_{i}$ and $P_{i}$ were provided by Martin [2]. The values for $\mu_{e n} / \varrho$ were interpolated from the data of Hubbell [3].

\begin{tabular}{c|c|c|c}
\hline \hline $\begin{array}{c}E_{i} \\
(\mathrm{keV})\end{array}$ & $100 P_{i}$ & $\begin{array}{c}\left(\mu_{e n} / \varrho\right) \times 10^{3} \\
\left(\mathrm{~m}^{2} / \mathrm{kg}\right)\end{array}$ & $\begin{array}{c}\dot{X}_{i} l^{2} \\
\left(\mu \mathrm{R} \cdot \mathrm{m}^{2} / \mathrm{s}\right)\end{array}$ \\
\hline 9.00 & 1.47 & 585 & 4.20 \\
9.44 & 4.0 & 530 & 10.86 \\
61.49 & 1.17 & 3.10 & 0.12 \\
63.00 & 2.02 & 2.78 & .19 \\
65.12 & 2.63 & 2.70 & .25 \\
66.83 & 4.52 & 2.66 & .44 \\
71.40 & 0.87 & 2.57 & .09 \\
75.70 & 1.97 & 2.48 & .20 \\
136.34 & 0.181 & 2.43 & .03 \\
201.31 & 0.466 & 2.67 & .14 \\
205.79 & 3.29 & 2.69 & .99 \\
283.26 & 0.261 & 2.85 & .11 \\
295.95 & 28.96 & 2.87 & 13.34 \\
308.45 & 29.67 & 2.88 & 14.30 \\
316.50 & 82.84 & 2.89 & 41.11 \\
374.48 & 0.729 & 2.94 & 0.44 \\
416.46 & 0.662 & 2.96 & .44 \\
468.06 & 47.8 & 2.96 & 35.93 \\
484.56 & 3.16 & 2.97 & 2.47 \\
489.06 & 0.397 & 2.97 & 0.31 \\
588.57 & 4.52 & 2.95 & 4.26 \\
604.40 & 8.18 & 2.95 & 7.91 \\
612.45 & 5.33 & 2.95 & 5.22 \\
884.52 & 0.302 & 2.84 & 0.41 \\
\hline
\end{tabular}

where: $\dot{X}$ is the exposure rate at distance $l$ from a point source

$A$ is the activity of the source

$W / e$ is the mean energy expended per unit charge in air $(33.7 \mathrm{~J} / \mathrm{C})$

$E_{i}$ is the photon energy

$P_{i}$ is the fraction of the nuclear transitions giving rise to photons of energy $E_{i}$

$\left(\mu_{e n} / \varrho\right)_{i}$ is the mass energy-absorption coefficient for photons of energy $E_{i}$

The current per unit volume of air, produced by an individual iridium-192 source of $1.5 \mathrm{mCi}$ activity, distant $1 \mathrm{~m}$ from an ionization chamber, is about $7 \times 10^{-17} \mathrm{~A} \cdot \mathrm{cm}^{-3}$. This is at least an order of magnitude less than the inherent leakage current for good electrometers. For accurate current measurements, therefore it is necessary to optimize the measurement conditions. This can be done by increasing the activity (by measuring a group of sources), using the largest-volume ionization chamber that is suitable, and using short measurement distances. The constraints imposed by the largest suitable chamber volume (approximately 50 $\mathrm{cm}^{3}$ ), and a reluctance to reduce the source-to-chamber distance to less than about one-half meter, led to the fabrication of two plane source arrays consisting of large numbers of seeds. The seeds were held in shallow recesses $(1 \mathrm{~cm}$ by 1
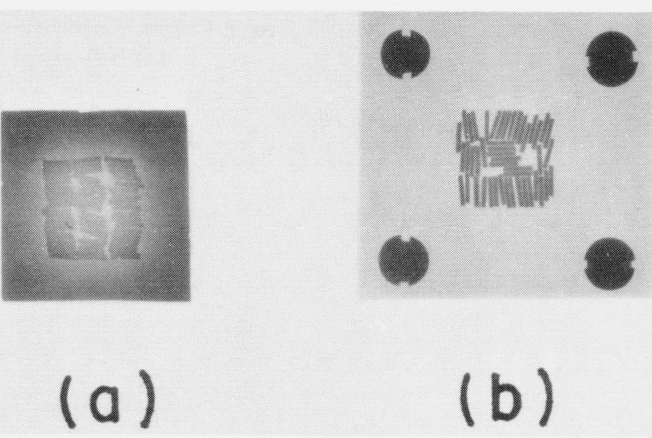

FIGURE 1. Radiographs of iridium-192 source arrays consisting of (a) 53 platinum-encapsulated sources and (b) 46 stainless-steel-encapsulated sources.

$\mathrm{cm}$ ) between thin pieces of plastic. The type and size of the two plastic source holders were slightly different (see fig. 1). One source array consisted of 53 of the platinum encapsulated seeds while the array for the stainless steel type consisted of 46 seeds. $^{2}$ Radiographs taken before and after all the measurements showed that the individual seeds had not changed position despite considerable handling.

\section{Standard Cavity lonization Chambers for Exposure Measurements}

The standard chambers employed for these measurements have already been described in detail [4]. Of the eight chambers available, the three with the largest air volumes (all nominally $50 \mathrm{~cm}^{3}$ ) were used. For some measurements, individual chambers were used, while for others, all three chambers were used. The equation for exposure computations is given in [4] but for ease of reference is also given here.

$$
X=\frac{Q_{a i r}}{v \varrho}(\bar{S} / \varrho)_{a i r}^{C}\left(\bar{\mu}_{e n} / \varrho\right)_{C}^{a i r} \Pi k_{i}
$$

where $X$ is the exposure ${ }^{3}, Q_{\text {air }}$ is the measured charge, $v$ is the chamber volume, and $\varrho$ is the density of air at the time of measurement. The ratio of the mean collision mass stopping powers for electrons in the medium (in this case graphite) and in the gas (air), $(\bar{S} / \varrho)_{a i r}^{C}$, must be calculated with consideration of the electron spectra produced by the many photon energies of iridium-192, as well as for the SpencerAttix parameter $\Delta$, which is the upper limit for electron energy losses and the lower limit on electron energies in-

\footnotetext{
${ }^{2}$ The Rad-Irid Co. supplied 49 seeds. However, the seeds were magnetized and could not be made to lie in an orderly plane array. Most of the magnetism was erased using a computer tape eraser but some residual magnetism limited to 46 the number of seeds that could be readily placed in the recess.

${ }^{3}$ The SI units for $X$ are coulombs per kilogram of air. To convert to roentgens divide by $2.58 \times$ $10^{-4}$.
} 
cluded in the spectrum. The value of $\Delta$ for the $50 \mathrm{~cm}^{3}$ standard chamber is taken to be $50 \mathrm{keV}$.

The mean stopping powers, for carbon and air, were determined by computing the initial Compton electron spectra [5] produced by each of the iridium-192 gamma-ray energies, weighting and summing the spectra in accord with the relative numbers of photons at each energy per disintegration, and then weighting and summing the stopping powers over the resultant electron energy spectrum. Stopping powers for a range of electron energies are given in table 3 . The ratio of the weighted mean stopping powers for carbon and air is 1.015 .

TABLE 3. Electron stopping powers calculated using the expression of Berger and Seltzer [6] for the restricted mean collision loss, with $\Delta=50 \mathrm{keV}, I_{C}=78 \mathrm{eV}$ and $I_{\text {air }}=86.8 \mathrm{eV}$. The data for carbon are corrected for the density effect, which is assumed negligible for air.

\begin{tabular}{c|c|c|c}
\hline \hline \multicolumn{3}{|c}{ Restricted collission mass stopping power $(\mathrm{S} / \mathrm{\varrho})$} \\
\hline $\begin{array}{c}\text { Electron energy } \\
(\mathrm{keV})\end{array}$ & $\begin{array}{c}\text { Carbon } \\
\left(\mathrm{MeV} \cdot \mathrm{cm}^{2} / \mathrm{g}\right)\end{array}$ & $\begin{array}{c}\text { Air } \\
\left(\mathrm{MeV} \cdot \mathrm{cm}^{2} \mathrm{~g}\right)\end{array}$ & $\begin{array}{c}\text { Ratio } \\
(\mathrm{S} / \mathrm{Q})_{\text {air }}^{C}\end{array}$ \\
\hline 60 & 6.091 & 5.998 & 1.016 \\
100 & 3.688 & 3.627 & 1.017 \\
150 & 2.831 & 2.790 & 1.015 \\
200 & 2.403 & 2.373 & 1.013 \\
250 & 2.147 & 2.123 & 1.011 \\
300 & 1.977 & 1.959 & 1.009 \\
350 & 1.858 & 1.844 & 1.008 \\
400 & 1.769 & 1.759 & 1.006 \\
450 & 1.702 & 1.695 & 1.004 \\
500 & 1.650 & 1.646 & 1.002 \\
550 & 1.608 & 1.606 & 1.001 \\
600 & 1.574 & 1.575 & 0.999 \\
650 & 1.546 & 1.550 & 0.998 \\
700 & 1.522 & 1.529 & 0.996 \\
750 & 1.503 & 1.512 & 0.994 \\
800 & 1.486 & 1.497 & 0.993 \\
850 & 1.472 & 1.485 & 0.991 \\
900 & 1.460 & 1.475 & 0.989 \\
\hline
\end{tabular}

Other than the experimental corrections included in the product $\Pi k_{i}$ which are discussed later, the remaining quantities to be determined are the mass energy-absorption coefficients $\left(\mu_{e n} / \varrho\right)$ for air and carbon. The data of Hubbell were used to determine the ratios of these coefficients which were then plotted against photon energy. Again, weighting factors (with consideration for attenuation in the seed wall) were used to compute values for $\left(\bar{\mu}_{e n} / \varrho\right)_{a i r} /\left(\bar{\mu}_{e n} / \varrho\right)_{C}$ of 1.001 and 1.002 , for the photon energies from the platinum and stainless steel encapsulated sources respectively.

\section{Experimental Corrections}

The corrections forming the product $\Pi k_{i}$ are of two kinds: those relating to the particular standard graphite ionization chamber, and those that take into consideration the sourceto-standard-chamber geometries and standard-chamber-toroom geometry. The various corrections are listed in table 4.

TABLE 4. Summary of corrections used in open-air geometry measurements of iridium-192 sources.

\begin{tabular}{clc}
\hline \hline Correction & Symbol & Factor \\
\hline $\begin{array}{l}\text { Wall absorption correction for } 50-1 \text { standard } \\
\text { graphite cavity ionization chamber for plat- }\end{array}$ & $k_{w}(\mathrm{Pt})$ & 1.028 \\
$k_{w}(\mathrm{Fe})$ & 1.033
\end{tabular}

inum and stainless steel encapsulated seeds

Recombination correction for 50-1 standard

$k_{s}$ 1.002 at 500 volts collecting potential for exposure rates of 10 to $36 \mu \mathrm{R} \cdot \mathrm{s}^{-1}$

Stem scatter correction

$k_{s s}$

0.999

Humidity correction

$k_{h}$

1.000

Room surface scatter corrections for $0.5-\mathrm{m}$

and $1.0 \mathrm{~m}$ measurements

$k_{r s}(0.5)$

0.993

$k_{r s}(1.0)$

0.972

Air attenuation-scattering corrections for $0.5-\mathrm{m}$

$k_{a s}(0.5)$

0.999

and $1.0-\mathrm{m}$ measurements

$k_{a s}(1.0)$

0.998

Attenuation correction for plastic enclosing iridium-192 source array

(Pt encapsulated)

(stainless-steel encapsulated)

$k_{p} \quad 1.005$

$k_{p} \quad 1.006$

The standard chamber wall-absorption correction was determined by positioning the plane sources in a collimatorshutter system to allow measurements with different standard chambers, and also to allow the addition of shells to the chambers. This arrangement avoided the need to move the source for protection purposes. The two techniques provided data that, when extrapolated to zero wall thickness, give wall corrections which agree to 0.1 percent. The average values of these corrections for the platinum-clad and steel-clad seeds are given in table 4 .

The exposure rates encountered in these measurements are relatively low and only moderately high collection potentials for the ionization chambers are required to minimize recombination effects. Calculations for the $50 \mathrm{~cm}^{3}$ standard chamber, based on previous data [4] for a collection potential of $500 \mathrm{~V}$, predict a correction factor of 1.0013 for the expected exposure rates. Checks were made on the chamber saturation conditions during the open-air geometry measurements by reducing the potential from $500 \mathrm{~V}$ to $250 \mathrm{~V}$. Results show a reduction in ionization current of 0.17 percent when the exposure rate is $9.3 \mathrm{nA} / \mathrm{kg}(36 \mu \mathrm{R} / \mathrm{s})$.

The standard chamber stem-scatter correction was determined by placing a dummy stem on the opposite side of the chamber stem in the collimated beam. The beam was large 
enough in diameter so as to irradiate the system uniformly but not so large as to irradiate the supporting stands. This mirror-image experiment provides a stem scatter correction of 0.999 .

All standard chamber ionization current data are corrected for atmospheric effects in accord with the ideal gas laws. The influence of water vapor on the measurements as it may affect the air density and the stopping-power ratios has not been taken into account and the correction for humidity is taken to be unity.

Source measurements in an open-air geometry require corrections for scattering from the room surfaces to make the measurement independent of the surroundings, and for air attenuation and scattering to make the source measurements independent of source-detector distance. The magnitude of these corrections depends on the various source-towall-to-chamber distances and source-to-chamber distances.

The dimensions of the measurement room are $6.0 \mathrm{~m}$ by $9.4 \mathrm{~m}$ by $4.4 \mathrm{~m}$ high. The source-chamber center line was vertical and roughly at the center of the room. Using the method of Eisenhauer [7], the scattering contribution from each surface of the room was calculated based on an albedo measurement. The measurements show that the concrete floor increases the chamber reading by about 16 percent, when the source is on the concrete floor, over that measured when the source and chamber are suspended in the center of the room with the source-chamber distance being one meter in each case.

Two source-to-chamber distances were used for the measurements, $0.5 \mathrm{~m}$ and $1.0 \mathrm{~m}$. The room surface scattering correction factors, $k_{r s}$, for the $1.0-\mathrm{m}$ and $0.5-\mathrm{m}$ measurements, are calculated to be 0.972 and 0.993 respectively.

The remaining correction is the air attenuation-scattering correction, the work of Eisenhauer [8] being used for the computations. The corrections, $k_{a s}$, for a photon energy range appropriate to iridium-192 and for $0.5-\mathrm{m}$ and $1.0-\mathrm{m}$ distances are given in table 5 . Mean values for $k_{a s}$, weighted

TABLE 5. Air attenuation-scattering correction factors, $k_{a s}$ in the photon energy range 0.2-0.5 MeV for two source-to-chamber distances.

\begin{tabular}{c|c|c}
\hline \hline \multirow{2}{*}{$\begin{array}{c}\text { Photon Energy } \\
(\mathrm{MeV})\end{array}$} & $0.5 \mathrm{~m}$ & $1.0 \mathrm{~m}$ \\
\cline { 2 - 3 } & 0.997 & 0.994 \\
0.2 & 0.999 & 0.998 \\
0.3 & 1.000 & 0.999 \\
0.4 & 1.000 & 1.000 \\
0.5 & & \\
\hline
\end{tabular}

in accord with the numbers of photons for the principal iridium-192 photon energies, are given in table 4.

The way in which these corrections are used depends on the desired result. To make source calibrations independent of source-detector distance and surface-source-chamber distances, the measurements are multiplied by the $k_{a s}$ and $k_{r s}$ factors. In this way, the consistency of measurements at different distances can be tested and the calibration made independent of the environment. However, when a calibrated source is used in the field, one wants to know the exposure rate at some distance from the source. Then the calibration for the source must be divided by the appropriate attenuation-scattering factor as well as the appropriate surfacescattering factor.

\section{Source Measurements}

The platinum-encapsulated seeds were measured in the open-air geometry at different times and different distances. Although nine measurements were performed, only five can be considered independent - three at approximately $0.5 \mathrm{~m}$ and two at approximately $1.0 \mathrm{~m}$ from the source. All ionization current data were corrected for decay using a half-life of 74 days $\left(\lambda=6.5 \times 10^{-6} \mathrm{~min}^{-1}\right)$ [2]. Two measurement systems were employed, a vibrating-reed-electrometer null method and a high-gain direct-coupled electrometer with a digital voltmeter for measurement of feedback potentials. The latter system allows automatic control of the digital voltmeter for initial and final measurements over preset charge-integration intervals. The built-in feedback capacitor in the high-gain electrometer was replaced with a calibrated air capacitor. The agreement among the various measurements is shown in table 6. The consistency of the measurements when corrected for scattering is demonstrated by the ratio of $\left(I D^{2} k_{r s} k_{a s}\right)$ for 0.5 and $1.0 \mathrm{~m}$ which is 1.005 , with the corrections and measurements data taken from tables 4 and 6 , respectively.

TABLE 6. Summary of independent source measurements in the open-air geometry method, platinum-clad sources.

\begin{tabular}{c|c|c|c|c}
\hline \hline $\begin{array}{c}\text { Source- } \\
\text { chamber } \\
\text { distance } \\
(\mathrm{m})\end{array}$ & $\begin{array}{c}\text { Current per } \\
\text { unit volume at } \\
\text { STP on } \\
\text { reference date } \\
\left(\mathrm{fA} / \mathrm{cm}^{3}\right)\end{array}$ & $\begin{array}{c}\text { Current } \\
\text { corrected to } \\
0.5 \text { and } 1.0 \mathrm{~m} \\
\left(\mathrm{fA} / \mathrm{cm}^{3}\right)\end{array}$ & $\begin{array}{c}\text { Mean } \\
\text { Current }\end{array}$ & $\begin{array}{c}\text { Current } \\
\text { normalized } \\
\text { to mean } \\
\text { current }\end{array}$ \\
\hline 0.4656 & 13.783 & 11.949 & & 0.999 \\
0.4612 & 14.044 & 11.946 & 11.966 & 0.998 \\
0.4709 & 13.530 & 12.003 & & 1.003 \\
0.9628 & 3.268 & 3.030 & 3.044 & 0.995 \\
0.9740 & 3.224 & 3.058 & & 1.005 \\
\hline
\end{tabular}

Although the number of measurements for the two distances was not large, weighting of the data in favor of the $0.5 \mathrm{~m}$ measurement seems justified. The usual weighting procedure (inverse of the variance of the mean) was used. The weighted mean current per unit volume was calculated 
to be $2.966 \times 10^{-15} \mathrm{~A} \cdot \mathrm{cm}^{-3}$ for the array of platinum-encapsulated sources at a distance of one meter.

Using eq (2) for $X$ and the data taken from tables 4 and 6 , the exposure rate at one meter from the platinum-encapsulated source array was calculated to be $2.411 \mathrm{nA} \cdot \mathrm{kg}^{-1} \cdot \mathrm{m}^{2}$ $\left(9.346 \mu \mathrm{R} \cdot \mathrm{s}^{-1} \cdot \mathrm{m}^{2}\right)$ on the reference date.

The stainless-steel-encapsulated source array was measured with the standard graphite chamber in the open-air geometry on two consecutive days; however, at only one source-to-chamber distance, about $44 \mathrm{~cm}$. With correction for decay and adjustment to a common source-chamber distance, the difference between the two measurements was 0.4 percent. The exposure rate at one meter was 0.5614 $n \mathrm{~A} \cdot \mathrm{kg}^{-1} \cdot \mathrm{m}^{2}\left(2.176 \mu \mathrm{R} \cdot \mathrm{s}^{-1} \cdot \mathrm{m}^{2}\right)$ on the reference date.

\section{Re-Entrant lonization Chamber}

A re-entrant ionization chamber, for measurement of individual iridium-192 seeds, was designed and fabricated at NBS. A drawing of the chamber is shown in figure 2. Originally a conducting plastic sphere was used for the outer shell but it was found to be out-gassing and to allow some electrostatic effects to be observed. Therefore it was replaced with an aluminum sphere as shown.

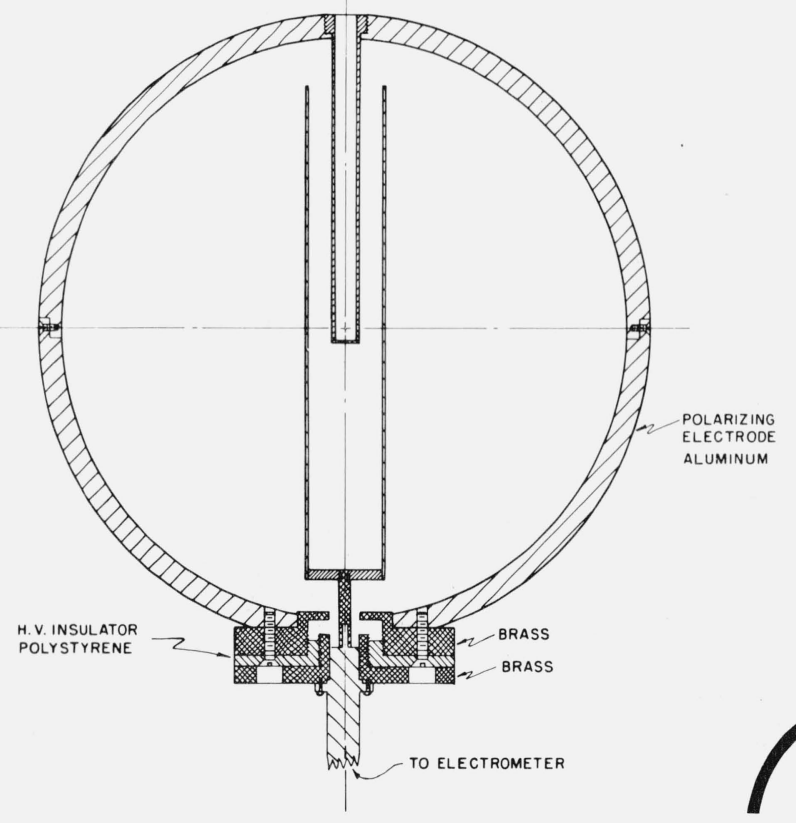

FIGURE 2. Aluminum re-entrant ionization chamber used for measurements of individual iridium seeds. The outside diameter of the aluminum sphere is $203 \mathrm{~mm}$.

Design considerations in determining the volume for the re-entrant chamber were based on an expected activity of about $1.5 \mathrm{mCi}$ for the iridium-192 sources and a knowledge that currents of the order of $5 \mathrm{pA}$ can be measured with a standard deviation of 0.1 percent, based on four measurements with charge integrating times of about $30 \mathrm{~s}$.

The current produced by a $1.5-\mathrm{mCi}$ iridium-192 seed in the aluminum sphere was $55.5 \mathrm{pA}$, while the same seed in the conducting plastic sphere, of essentially the same volume, produced $49.0 \mathrm{pA}$. The 13 percent increase in current is due to the energy dependence of chamber response when an air-equivalent material is replaced by aluminum as the wall of the ionization chamber.

The collection electrode is a hollow cylinder surrounding the re-entrant tube. It is guarded and the high voltage insulator is recessed. The chamber is usually operated at a potential of $1100 \mathrm{~V}$ on the aluminum sphere, with a positive ground. Since the sphere is at high potential it is necessary to use an insulating holder for sources placed in the chamber. Glass vials of length $65 \mathrm{~mm}$, with an outer diameter of $9.2 \mathrm{~mm}$ and wall thickness of $1.4 \mathrm{~mm}\left(0.29 \mathrm{~g} / \mathrm{cm}^{2}\right)$ are found to be convenient for this purpose. A thin plastic tube is used to insert the vials into the chamber. The internal diameter of the vials is such that the 3 mm-long iridium-192 sources lie almost horizontally at the bottom of the vials.

The recombination characteristics for the aluminum sphere, assuming a linear response proportional to the inverse of the collection potential, are such that the correction for ionization loss is 0.36 percent for currents of $6.6 \mathrm{pA}$ to $76 \mathrm{pA}$ at the operating collection potential of 1100 volts. The percentage loss increases to 0.6 percent for a current of $884 \mathrm{pA}$ for the same collection potential. In these tests, the lowest current was produced by an iridium-192 source while the two higher currents were produced by cesium-137 sources.

The polarity dependence of the chamber is characteristic of the collection electrode-insulator system and was found to be the same for the plastic and aluminum spheres. The ratio of the chamber currents measured with positive ground, to currents measured with negative ground, is 1.0014 .

\section{Iridium-192 Seed Measurements in Re-Entrant Chamber}

Although the plastic re-entrant chamber was replaced with the aluminum chamber, and it will not be used routinely for calibrations, it is of interest to determine the degree of agreement between relative measurements of the seeds, in each chamber, for different measurement conditions. The comparison can be made through measurements of the 53 platinum-encapsulated seeds which were carried out in each of the chambers.

Each of the seeds was placed in a glass vial and measured in each of the re-entrant chambers. The measurements using the plastic sphere were carried out in eight hours while the measurements using the aluminum sphere were com- 
pleted in approximately four hours. In each case, the data were corrected for decay to the same time. The consistency of the source measurements using the two chambers was tested by summing the currents and determining the fraction of the total current contributed by each seed. The standard deviation of the ratios of the seed calibrations for these two sets of measurements is 0.2 percent with one outlier of 1.2 percent included.

The calibration factors for the re-entrant chambers are the quotients of the exposure rate, for the seed array in the open-air measurement, and the sum of the individual currents produced by the seeds in the re-entrant chambers. The factors for the plastic and aluminum spheres determined for the platinum-encapsulated seeds, as well as the factor for the stainless-steel-encapsulated seeds in the aluminum chamber, are given in table 7 . The calibration factor determined for the stainless-steel-encapsulated sources in the aluminum chamber is 3 percent different from that determined for the platinum-encapsulated sources.

Because of the relatively short half-life of iridium-192, the reference for exposure calibrations for this isotope will be the re-entrant chamber and its calibration factors. Although the chamber design is such that no problem with constancy is foreseen, a $0.5 \mathrm{mg}$ radium source, in a special plastic thistle tube for handling, has been used as an overall reference source throughout the measurements. This source will be used to insure the consistency of all iridium source measurements in the future.

TABLE 7. Summary of re-entrant chamber calibration data.

\begin{tabular}{|c|c|c|c|c|c|}
\hline \multirow[t]{2}{*}{$\begin{array}{c}\text { Source } \\
\text { encapsulation }\end{array}$} & \multirow[t]{2}{*}{ Chamber } & \multirow{2}{*}{$\begin{array}{c}\text { Exposure rate } \\
\text { of array } \\
\left(\mathrm{nA} \cdot \mathrm{kg}^{-1} \cdot \mathrm{m}^{2}\right)\end{array}$} & \multirow{2}{*}{$\begin{array}{c}\text { Total } \\
\text { current } \\
\text { (nA) }\end{array}$} & \multicolumn{2}{|c|}{ Calibration factors } \\
\hline & & & & $\left(\mathrm{kg}^{-1} \cdot \mathrm{m}^{2}\right)$ & $\left(\mathrm{k} R \cdot \mathrm{s}^{-1} \cdot \mathrm{A}^{-1} \cdot \mathrm{m}^{2}\right)$ \\
\hline Platinum & Plastic & 2.411 & 2.438 & 0.9889 & 3.833 \\
\hline " & Aluminum & 2.411 & 2.831 & 0.8516 & 3.301 \\
\hline Stainless steel & Aluminum & 0.5614 & 0.6779 & 0.8281 & 3.209 \\
\hline
\end{tabular}

\section{Assessment of Uncertainties}

The factors which influence the results of the exposure measurements, along with their estimated uncertainties, are listed in table 8. The statistical uncertainties given are three times the standard deviation of the mean, while the systematic uncertainties are estimated upper bounds.

The statistical uncertainty for the long-term reproducibility of the measurements deserves special comment. To arrive at the value given in table 8 , the data from table 6 were corrected using the values for $k_{r s}$ and $k_{a s}$ and then corrected to a common distance via the inverse square law. The standard deviation of those five $a b$ initio measurements is 0.5 percent and the standard deviation of the mean is 0.2 percent. The value given in table 8 is three times the standard deviation of the mean of the five measurements. Although statistical effects of current and distance measurements influence these data, they do not significantly affect the outcome. While the differences between the means of the corrected data for $0.5 \mathrm{~m}$ and $1.0 \mathrm{~m}$ measurements indicate a possible inadequacy in the corrections for attenuation and scattering, there are not enough measurements to support this conclusion and this difference can be either statistical or systematic.

The quadratic sum of the systematic uncertainties is 0.7 percent, and like treatment of the statistical uncertainties gives 0.6 percent. If all of these uncertainties are added in quadrature an estimate of the overall uncertainty is 1 per-
TABLE 8. Estimated uncertainties in the determination of the exposure rate at one meter from an iridium-192 seed.

\begin{tabular}{|c|c|c|}
\hline & \multicolumn{2}{|c|}{ Uncertainty $\mu(\%)$} \\
\hline & Systematic & Statistical \\
\hline \multicolumn{3}{|l|}{ Standard chamber: } \\
\hline Air volume & 0.02 & \\
\hline \multicolumn{3}{|l|}{ Corrections: } \\
\hline Wall Absorption & 0.1 & \\
\hline Stopping power & 0.5 & \\
\hline Energy absorption & 0.3 & \\
\hline Recombination & 0.02 & \\
\hline Stem scatter & 0.02 & \\
\hline \multicolumn{3}{|l|}{ Scattering: } \\
\hline Room surfaces & 0.3 & \\
\hline Air attenuation and scatter & 0.1 & \\
\hline \multicolumn{3}{|l|}{ Current at one meter (open-air): } \\
\hline Current & 0.03 & 0.1 \\
\hline Humidity & 0.3 & \\
\hline Temperature & 0.03 & \\
\hline Pressure & 0.01 & \\
\hline Distance & & 0.01 \\
\hline Reproducibility (long-term) & & 0.6 \\
\hline \multicolumn{3}{|l|}{ Re-entrant chamber: } \\
\hline Current & 0.03 & \\
\hline Fraction current & & 0.1 \\
\hline Total current & & 0.15 \\
\hline Temperature & 0.03 & \\
\hline \multirow[t]{2}{*}{ Pressure } & 0.01 & \\
\hline & $\sqrt{\Sigma} u^{2}=0.7$ & $\sqrt{\Sigma u^{2}}=0.6$ \\
\hline
\end{tabular}


cent. But since this is a new calibration service, and in the absence of checks from other laboratories, we propose to take the overall uncertainty associated with an iridium seed calibration as 2 percent.

\section{Addendum}

After the work described in this article was complete the Radiological Physics Center at M.D. Anderson Hospital in Houston requested exposure calibrations of platinumencapsulated iridium-192 wires which were $10 \mathrm{~mm}$, and 50 $\mathrm{mm}$, long. All other dimensions of the wires were the same as described in table 1 . Since the calibration factors for the re-entrant chamber had been determined for seeds which lay almost horizontally in the re-entrant tube and the longer wires would stand almost vertically, it was necessary to determine a new re-entrant chamber calibration factor for this condition.

Nine 10-mm-long wires and two 50-mm-long wires of high specific activity were provided by the Radiological Physics Center. The $10-\mathrm{mm}$ wires were immobilized in the plastic holder used for the stainless-steel seed measurements, and open-air measurements using one of the standard graphite ionization chambers were carried out as already described. The exposure rate from this group of sources on the reference date was determined to be $2.051 \mathrm{nA} \cdot \mathrm{kg}^{-1} \cdot \mathrm{m}^{2}(7.951$ $\left.\mu \mathrm{R} \cdot \mathrm{s}^{-1} \cdot \mathrm{m}^{2}\right)$.

The wires were removed from the plastic holder and each wire was measured individually in the re-entrant chamber. The sum of the currents, corrected for recombination and corrected to the reference date, was $2416 \mathrm{pA}$. The quotient of the open-air group exposure rate, and the total re-entrant chamber current, is the aluminum re-entrant chamber calibration factor for 10-mm-long platinum seeds. The calibration factor is $0.8489 \mathrm{~m}^{2} \cdot \mathrm{kg}^{-1}$ and, in special units, is 3.290 $\mathrm{kR} \cdot \mathrm{s}^{-1} \cdot \mathrm{m}^{2} \cdot \mathrm{A}^{-1}$. Thus, the re-entrant chamber response for the 1 -cm-long wires in the upright position is 1 percent greater per unit exposure than the response for the 3-mmlong seeds.

The dependence of the re-entrant chamber response on the length of the iridium-192 wire was found by measuring the current produced by one of the 10-mm-long sources as it was raised in 10-mm increments up the re-entrant tube. A thread affixed to the end of the wire was used for this purpose. Correction factors relative to the re-entrant chamber calibration factor for 10-mm-long iridium-192 wires are given in table 9 .

TABLE 9. Iridium-192 wire-length correction factors relative to the aluminum re-entrant chamber calibration factor for a 10-mm-long wire.

\begin{tabular}{c|c}
\hline \hline Length $(\mathrm{mm})$ & Correction factor \\
\cline { 2 - 2 } 10 & 1.000 \\
20 & 1.004 \\
30 & 1.008 \\
40 & 1.013 \\
50 & 1.019 \\
60 & 1.026 \\
\hline
\end{tabular}

I wish to thank Mr. George Harwood of the Alpha-Omega Co., Mr. K. Sunthanthrian of the Rad Irid Co., and Messrs. Jack Cundiff and Will Hanson of the Radiological Physics Center at M.D. Anderson Hospital, for their cooperation in this project and for providing the iridium-192 sources required for the measurements.

I also wish to thank R. Loevinger for his critical comments and editorial help in preparing this article for publication.

\section{References}

[1] A Manual of Radioactivity Measurements and Procedures, Nat. Council on Radiation Protection and Measurements Report No. 58. P.0. Box 30175, Washington, DC 20014.

[2] Martin, M., Nuclear Data Project, ORNL. Oak Ridge, Tennessee 37830. (Priv. Comm. May 1977)

[3] Hubbell, J. H., Photon Mass Attenuation and Mass Energy-Absorption Coefficients for H, C, N, O, Ar, and Seven Mixtures from $0.1 \mathrm{keV}$ to $20 \mathrm{MeV}$. Radiat. Res. 70 58-81 (1977).

[4] Loftus, T. P. and Weaver, J. T., Standardization of ${ }^{60} \mathrm{Co}$ and ${ }^{137} \mathrm{Cs}$ Gamma-Ray Beams in Terms of Exposure. J. Res. Nat. Bur. Stand. (U.S.A.) Physics and Chemistry, 78A, No. 4 (July-August 1974).

[5] Nelms, A. T., Graphs of the Compton Energy-Angle Relationship and the Klein-Nishima Formula from $10 \mathrm{keV}$ to $500 \mathrm{MeV}$, National Bureau of Standards (U.S.) Circular 542, 89 pages, Aug. 28, 1953.

[6] Berger, M. J. and Seltzer, S. M., Tables of Energy Losses and Ranges of Electrons and Positrons, NASA SP-3012, 1964 (available from the Clearinghouse for Scientific and Technical Information, Springfield, Virginia).

[7] Eisenhauer, C., An Image Source Technique for Calculating Reflection of Gamma Rays or Neutrons, Health Physics 11, 1145-1154 (1965).

[8] Eisenhauer, C., A study of the Angular and Energy Distributions of Radiation at Small Distances from a Point Source of Gamma Rays or Neutrons, Nuclear Science and Engineering, 27, 240-251 (1967). 
